\title{
Reconstrucción preimplante con hueso autólogo de procedencia sinfisaria en la rehabilitación de defectos óseos transversales de los maxilares: protocolo a realizar en la consulta
}

\section{Pre-implant recostruction with autologous bone from mental symphysis during transverse maxillary bone atrophies rehabilitation: surgery protocol}

\author{
CARINI F* $* *, * * *, * * * *$ \\ PORCARO G*,*** \\ CIARAVINO IM* **** \\ MONAI D*, ** \\ FRANCESCONI M* \\ BALDONI IM*,**,***,****
}

\begin{abstract}
Carini F, Porcaro G, Ciaravino M, Monai D, Francesconi M, Baldoni M. Reconstrucción preimplante con hueso autólogo de procedencia sinfisaria en la rehabilitación de defectos óseos transversales de los maxilares: protocolo a realizar en la consulta. Av Periodon Implantol. 2009; 21, 1: 11-19.
\end{abstract}

\section{RESUIMEN}

Introducción: En los casos examinados, de atrofias de entidad moderada (IV clase de Cawood y Howell) (1), se ha observado un espesor transversal de la cresta inferior a $4 \mathrm{~mm}$, por tanto una rehabilitación implantosoportada resulta difícil de realizar.

Finalidad del trabajo: rehabilitación implantoprotésica de las atrofias de entidad moderada en los maxilares.

Materiales y métodos: 42 implantes realizados en 18 pacientes ( 8 varones y 10 mujeres, con una edad media de 47.1 años), en maxilar atrófico rehabilitado con la interposición de una muestra de hueso autólogo procedente de la sínfisis mentoniana. Los implantes han sido colocados a los 6 meses de haber aumentado transversalmente la cresta edéntula.

Se han valorado: la osteointegración, con niveles de pérdida ósea correspondientes, la condición de los tejidos perimplantarios, el índice de profundidad del saco, de placa y de sangrado, así como el grado de satisfacción del paciente.

Resultados: De 42 implantes no se perdió ninguno (0\%), una vez finalizada la fase de seguimiento. La pérdida de hueso marginal perimplantario ha sido, en media, de 0,17 mm.

Conclusiones: La utilización de muestras autólogas de zonas intraorales para el tratamiento de atrofias de entidad moderada ha demostrado ser una técnica con una predictibilidad elevada, que ha permitido efectuar implantes protésicos con un porcentaje de éxito elevado.

Consecuencias clínicas: Aunque este procedimiento prevé dos zonas quirúrgicas en el mismo acto quirúrgico, necesitándose, por tanto, una elevada implicación del paciente, es indiscutible la ventaja de poder intervenir sin anestesia total, con la ayuda, exclusivamente, de anestesia locorregional.

PALABRAS CLAVE: Atrofia ósea, injerto de hueso autólogo, sínfisis mentoniana.

\section{SUMIMARY}

Introduction: in the case studies the moderate atrophies (Cawood and Howell's $4^{\text {th }}$ class) is observed a transverse thickness lower than $4 \mathrm{~mm}$, therefore carring out a riabilitation by prosthesis appears very difficoult.

* Departamento de Cirugía Oral. University of Milano-Bicocca. San Gerardo University Hospital. Monza. Italy.

** Departamento de Cirugía Oral. Policlinico di Monza. Verano. Italy.

*** Departamento de Cirugía Oral. Don Gnocchi. I.R.C.C.S. Milano. Italy.

**** Departamento de Cirugía Oral. Ferb Foudation. Bergamo. Italy. 
Aims: Implanto-prosthesis rehabilitation of moderate maxillary atrophies (Cawood and Howell's $4^{\text {th }}$ class) (1).

Materials and Methods: 42 implants in 18 patients (8 males and 10 females, of medium age of 47.1 years), in an atrophic maxilla rehabilitated through interposition of autologous bone drawed from mental simphysis. The implantans were located after 6 months from the cross-sectional increase operation of edentulous crest.

We have considered: the osteointegration related to bony loss, the perimplant soft tissue condition, depth index of gingival pocket, plaque and bleeding, and even patients satisfaction index.

Results: at a complete follow-up no implants were lost. The mean of marginal perimplant bony loss was $0.17 \mathrm{~mm}$.

Conclusions: to manage moderate atrophies we have shown a technique with a good prognosis by using autologous drawings from intraoral centers, wich let successfull execution of implanto-prosthesic treatments.

Clinical implications: even if the tecnique involves two simultaneous surgery sites, needing an high compliance by the patient, is unquestionable the vantage to can operate without narcosis, only availing of local anesthesia.

KEY WORDS: Bone atrophy, autologous bone graft, chin symphysis.

Fecha de recepción: 14 de julio 2008.

Fecha de aceptación: 21 de julio 2008.

\section{INTRODUCCIÓN}

La atrofia es una condición que se caracteriza por una reducción de volumen del tejido óseo, ya completamente desarrollado, con la consiguiente insuficiencia funcional. La atrofia puede ser fisiológica, cuando estructuras ya completas evolucionan según su regresión natural, traumática o patológica.

La entidad de la atrofia puede ser de distinta importancia: para definir mejor los cuadros de atrofia más importantes que necesitan de cirugía preimplantaria, recordamos la clasificación de Cawood y Howell, propuesta en 1988, que ha sido reconocida y aceptada universalmente. En esta clasificación se distinguen 6 grupos: la primera clase, identifica una cresta alveolar edéntula normal, la segunda clase una cresta alveolar postextracción, la tercera clase, una cresta alveolar redondeada pero con un grosor suficiente y una altura tal que sus características no constituyen un problema ni protésico ni implantológico, la cuarta clase, una cresta alveolar denominada "en filo de cuchillo", con una altura adecuada (entre 7 y $10 \mathrm{~mm}$ ) pero con un espesor reducido, la quinta clase una cresta aplanada y por tanto con una altura y un espesor reducido, la sexta clase una pérdida total del hueso alveolar con pérdida incluso de hueso basal. Es especialmente importante para los autores el "managment" de las atrofias de IV clase.

\section{Biología de los injertos óseos}

En base a los estudios histológicos y clínicos realizados, se ha demostrado que el tejido óseo transplantado bajo forma de bloque de cortical, hueso corticoesponjoso, o sólo esponjoso bajo forma de bone chips para corregir la atrofia ósea, presenta distintas fases de reajuste e integración estandarizado en los tiempos y en los procesos biológicos. Numerosos estudios histológicos (Tabla 1) demuestran que los osteocitos y los osteoblastos presentes en el injerto óseo se someten a nuevo reajuste y se sustituyen completamente con el tejido óseo neoformado en un tiempo bien codificado, de tal manera que cuatro meses después del injerto nos encontramos en el momento ideal para realizar el implante, pues el tejido óseo, presentando una gran vitalidad, garantiza una mejor osteointegración.

En resumen, podemos recordar que, sin ninguna duda, el injerto se reabsorbe completamente, pues actúa como estructura (Scaffold) sobre la que se forma nuevo tejido óseo.

Gracias a los estudios histológicos realizados, ha sido posible comprender y afirmar que los implantes se introducen al final del periodo de reabsorción, cuando la infiltración inflamatoria ha desaparecido y cuando la actividad osteogenética y la neovascularización 


\section{TABLA 1.- ESQUEIMA DE LA BIOLOGÍA DE LOS INJERTOS ÓSEOS Y MODIFICACIONES CON LAS QUE SE VEN SOIMETIDAS A LO LARGO DEL TIEIMPO}

\section{Biología de los injertos óseos}

\begin{tabular}{|l|l|}
\hline 2 semanas & $\begin{array}{l}\text { Disminución del número de osteocitos vitales. } \\
\text { Infiltración inflamatoria alrededor del injerto consistente en neutrófilos polimorfonucleados y } \\
\text { linfocitos. } \\
\text { Iniciales signos de reabsorción en la periferia del injerto. } \\
\text { Inicio de la neo-vascularización. }\end{array}$ \\
\hline 1 mes & $\begin{array}{l}\text { Completa desaparición de los osteocitos vitales. } \\
\text { Persiste la infiltración inflamatoria alrededor del injerto. } \\
\text { Evidentes signos de reabsorción en las zonas periféricas del injerto, con signos de actividad } \\
\text { osteoclástica y neoformación de tejido osteoide. } \\
\text { Aumento de la neo-vascularización con amplio número de vasos neo-formados. }\end{array}$ \\
\hline 2 meses & $\begin{array}{l}\text { Neta disminución de la infiltración inflamatoria, que se encuentra presente sólo en algunas pe- } \\
\text { queñas zonas. } \\
\text { Reabsorción en fase muy avanzada, persisten todavía algunas zonas del injerto (pocas) no } \\
\text { revitalizadas. } \\
\text { Presencia de hueso vital neoformado y de tejido osteoide. }\end{array}$ \\
\hline 4 meses & $\begin{array}{l}\text { Desaparición de la infiltración inflamatoria. } \\
\text { Completa reabsorción, no persisten restos del injerto en los casos de injertos bajo forma de } \\
\text { bone chips; sigue su curso el proceso de reabsorción en caso de injertos cortico-esponjosos en } \\
\text { bloque único. } \\
\text { Eltejido óseo neoformado se presenta ya maduro, con intensa actividad osteoblástica y osteo- } \\
\text { clástica. } \\
\text { La vascularización llega al máximo grado de intensidad. }\end{array}$ \\
\hline 8 meses & $\begin{array}{l}\text { Presencia completa de hueso maduro, con disminución de la actividad osteoblástica y osteo- } \\
\text { clástica. } \\
\text { Disinución de la vascularización. } \\
\text { Si el tejido óseo no se estimula, empieza el proceso de reabsorción ósea. }\end{array}$ \\
\hline
\end{tabular}

son más intensas, y por tanto, como hemos visto, alrededor del cuarto mes. Introducir los implantes posteriormente, significa disponer de un tejido óseo injertado en reducción, biológicamente menos receptivo con los implantes que se deben osteointegrar.

\section{MATERIALES Y MÉTODOS}

El reconocimiento de dichas atrofias tiene lugar inicialmente con la inspección al examen objetivo, con la valoración de la ortopantomografía, con radiografías periapicales que se completarán, si es necesario, con una tomografía computarizada (Fig. 1) o, de otro modo, por observación directa en fase intraoperatoria.
El estudio postoperatorio del caso debe comprender, además, los modelos en escayola de las arcadas y las fotografías intraorales y extraorales del paciente (2).

La determinación de la entidad de la atrofia y de la zona donante más idónea, son un paso fundamental para el buen resultado de la intervención: los autores determinan la zona donante en función del volumen de la atrofia de la zona receptora. Para atrofias con un volumen superior a los 1,5 cc se elegirá una zona extraoral (cráneo; cresta ilíaca; tibia); para volúmenes inferiores se podrá recurrir, en cambio, a zonas intraorales como la sínfisis mentoniana, la tuberosidad maxilar o la rama mandibular. 


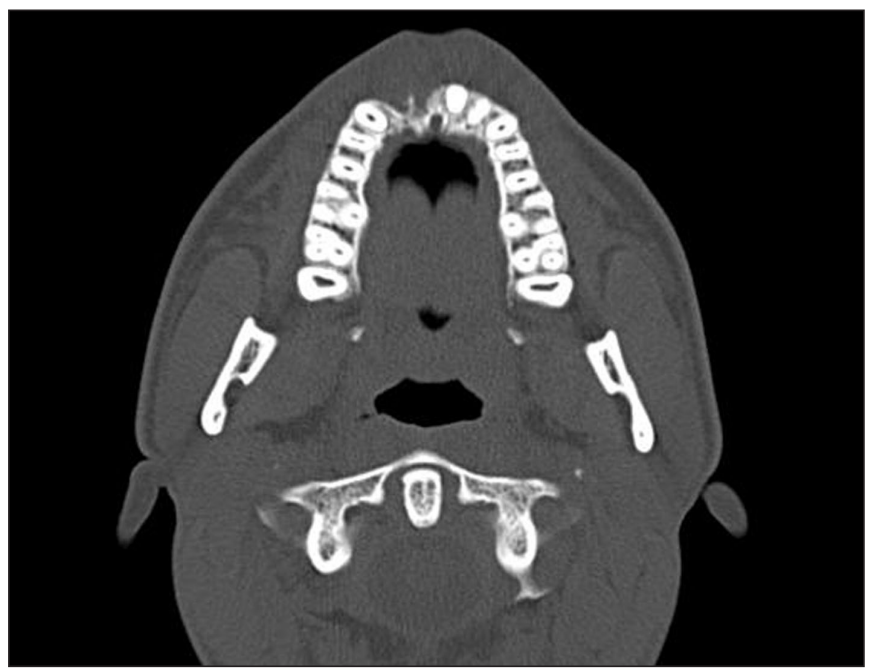

Fig. 1: Tomografías computarizadas preoperatoria.

Los autores prefieren como zona donante la sínfisis mentoniana:

- La intervención puede realizarse con anestesia local.

- Existe una sola zona operatoria (con consiguiente reducción de la morbilidad) (Misch y otros 1992; Misch 1997) (3, 4).

- Ausencia de cicatrices o deformidades visibles; se evitan problemas de deambulación y de movilidad articular (típicos de las extracciones extracraneales).

- A diferencia de las extracciones extracraneales, los injertos de origen intraoral presentan una menor tendencia a reabsorberse (Zins \& Whittacker 1983; «Phillips» \& Rhan 1990) $(5,6)$.

Además, se prefiere esta zona respecto a otras (tuberosidad maxilar y rama mandibular) por distintos motivos:

- Mayor accesibilidad.

- Falta de importantes estructuras vasculares-nerviosas en las inmediatas cercanías (nervio mandibular en el caso de la rama mandibular y nervio palatino mayor para el tuber maxillae).

- Menor sensibilidad postoperatoria.

El elemento menos positivo para esta zona es la forma de la sínfisis: en efecto, si el paciente presenta un mentón con dimensiones reducidas desde el punto de vista anatómico, la cantidad de hueso que se puede obtener será limitada; en ese caso habrá que valorar si es oportuno obtener una muestra de la sínfisis e integrar con un injerto heterólogo, o bien obtenerlo de una zona donante distinta.
El grupo de pacientes considerados en el estudio presentan una atrofia que se ha creado después de:

- Un traumatismo, con pérdida de elementos y de tejido óseo.

- Edentulismo prolongado.

El periodo de seguimiento del grupo de estudio es de 12 meses, una vez concluida la rehabilitación. En esta fase no deben manifestarse síntomas o indicios anómalos como:

- Infecciones.

- Movilización/reabsorción parcial o total del injerto (control con palpación manual y control radiológico).

- Pérdida del implante.

Los controles radiológicos consisten en una ortopantomografía y en una tomografía computarizada, que se efectúan inmediatamente antes e inmediatamente después de la intervención quirúrgica de introducción del injerto, a los $4 / 6$ meses y a los 12 meses de haber colocado la prótesis.

La participación de los pacientes en la experimentación está subordinada a algunos criterios de exclusión:

- Escasa higiene oral.

- Bruxismo.

- Fumador empedernido (más de 5 cigarrillos al día).

- Persona sometida a radioterapia en la zona cabeza-cuello en el último año.

- Persona sometida a quimioterapia.

\section{$1^{\text {ex }}$ estadio quirúrgico}

Se ha sometido al paciente a anestesia locorregional con carbocaína con adrenalina, a una concentración de 1/100.000; seguidamente, se ha realizado una incisión en cresta de grosor total, con extensión al surco gingival de los elementos adyacentes al espacio edéntulo. Se han realizado más tarde dos cortes de descarga para aumentar la movilidad del colgajo, sobre todo a nivel bucal. La exposición de la estructura subyacente permite confirmar el análisis preoperatorio realizado sobre la zona receptora (Fig. 2).

Se practica una osteoplastia de la zona donante de tal manera que quede una superficie más uniforme sobre la cual pueda agarrar el injerto. También en fase intraoperatoria se ha preparado un patrón con un folio 


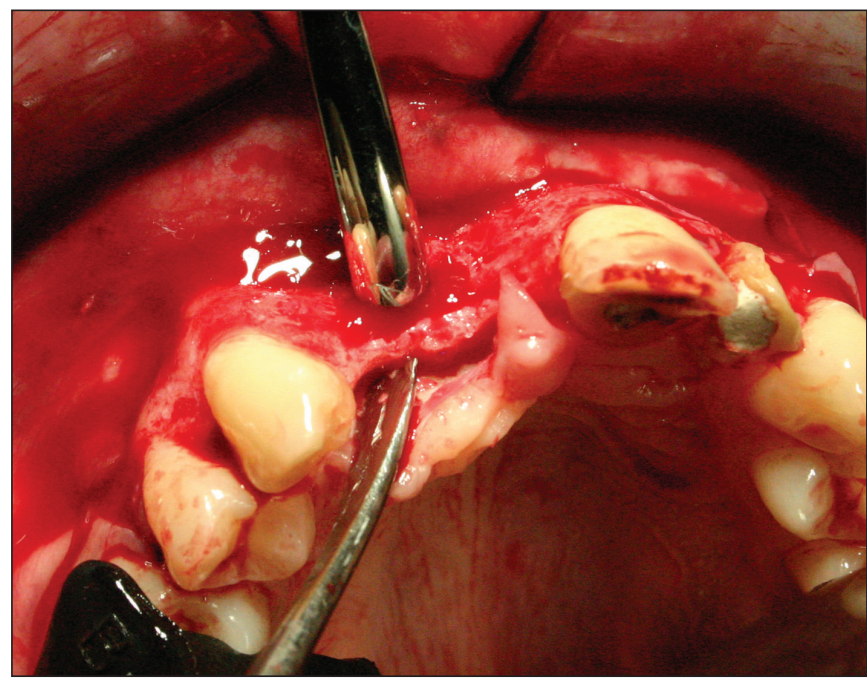

Fig. 2: imagen intraoperatoria de una atrofia de grado IV.

de aluminio, de tal manera que pueda efectuarse un dibujo preciso de la extracción.

Por lo que se refiere a la zona donante, el colgajo ha sido obtenido realizando con un corte horizontal en el vestíbulo, de canino a canino, con cortes de descarga para conseguir así un acceso más fácil. Una vez alcanzada la cortical ósea se ha procedido a la extracción utilizando instrumental rotatorio o con bisturí piezoeléctrico; en la mayor parte de los casos hemos utilizado éste último, pues permite cortar estructuras consistentes como hueso y dientes, evitando selectivamente los tejidos blandos (fig. 3).

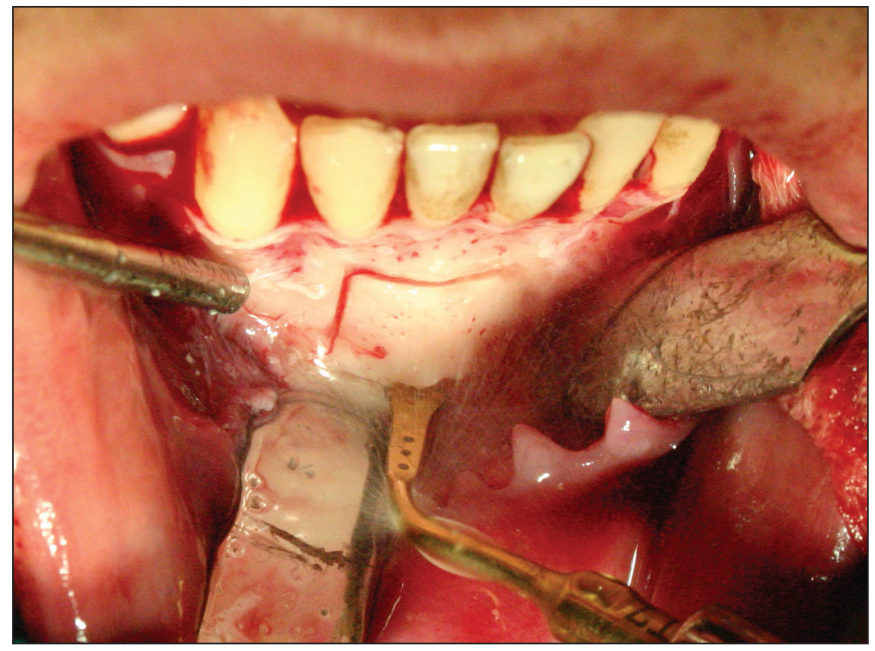

Fig. 3: Preparación del injerto con el bisturí piezoeléctrico.
Además, es más fácil controlar las distintas operaciones si se utilizan instrumentos rotatorios y cortantes, es menos traumático para el hueso y los tejidos implicados en la intervención no se contaminan, con una consiguiente reducción de la invasión del gesto quirúrgico.

El lugar del que obtener el injerto debe considerar la posición de los ápices radiculares de los elementos sobresalientes y por tanto, con la ayuda de la TAC, el primer corte se realiza al menos $3 \mathrm{~mm}$ por debajo de los ápices radiculares. Empezando por el primer lecho realizado, superponiendo el patrón a la cortical, se traza todo el perímetro del injerto y posteriormente se profundiza en los márgenes hasta llegar al espesor necesario para rellenar el defecto de la zona receptora. Para efectuar el desprendimiento del injerto utilizamos un pequeño escoplo de hueso, utilizado con una leve acción de cuña, colocándolo a una inclinación de $45^{\circ}$ en el margen del injerto, a lo largo del perímetro, hasta obtener la movilización definitiva.

$\mathrm{El}$ injerto se modela posteriormente para que pueda acoplarse perfectamente en la zona receptora y sucesivamente se procede a su transfixión mediante uno o varios tornillos de acero, comprobando al final la estabilidad del injerto y realizando la sutura (Fig. 4).

A nivel de la zona donante se controla la hemostasia mediante redes o bloques de nitrocelulosa oxidada, tras lo cual se ha procedido a la sutura.

En ambas zonas se utilizará seda, trenzada, de 4 ceros.

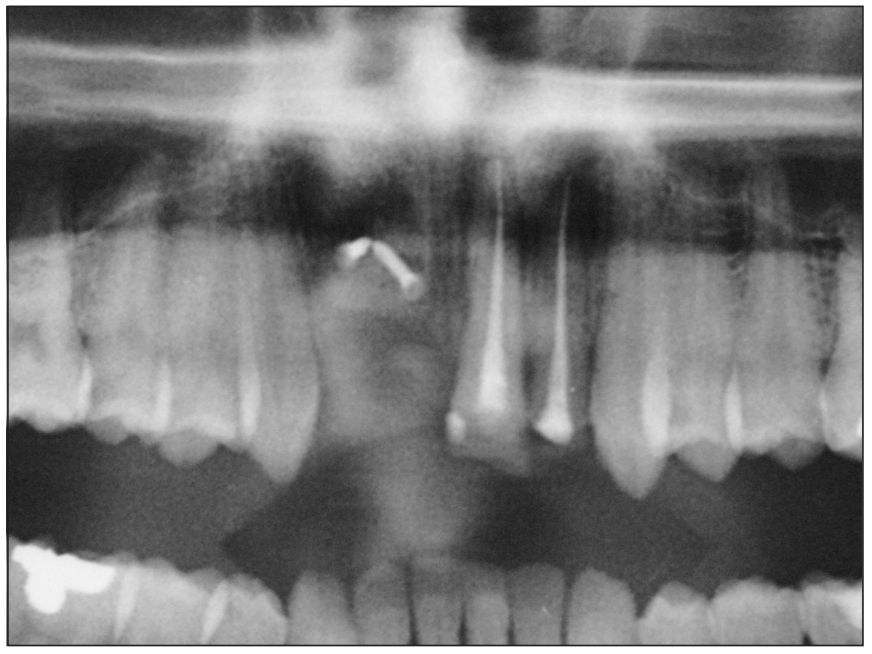

Fig. 4: Tornillo de fijación ósea. 
Inmediatamente después de la intervención se suministrarán $8 \mathrm{mg}$ de betametasona y $2 \mathrm{~g}$ de amoxicilina I.M. Se prosigue la terapia antibiótica durante 5 días con $1 \mathrm{~g}$ de amoxicilina cada 8 horas.

El paciente ha sido informado convenientemente sobre el uso de antiinflamatorios no esteroides, y en caso de dolor se le suministran $100 \mathrm{mg}$ de nimesulida cada 12 horas, durante 3 días, después de la intervención.

Se han prescrito enjuagues con colutorio a base de clorexidina al $2 \%$ por un minuto después de la higiene oral normal.

A las 2 semanas se procede a la extracción de los puntos, y en la misma sesión se vuelve a colocar la prótesis fija (puente Maryland) provisional.

\section{$2^{\circ}$ estadio quirúrgico}

Después de un periodo de recuperación de 6 meses, se colocan los implantes: se ha aplicado una anestesia locorregional de carbocaína con adrenalina a una concentración de 1/100.000; sucesivamente se ha efectuado una incisión en cresta de grosor total, con extensión al surco gingival de los elementos adyacentes al espacio edéntulo. Se han realizado también dos incisiones de descarga para aumentar la movilidad del colgajo, sobre todo a nivel bucal. Se han preparado las zonas afectadas por el implante. Se han introducido 18 implantes de $3,5 \mathrm{~mm}$ de diámetro, y 24 de 4 $\mathrm{mm}$ de diámetro, todos ellos de $10 \mathrm{~mm}$ de longitud. En total se han colocado 42 implantes. Seis meses más tarde se ha realizado la segunda intervención y se han colocado los tornillos de recuperación. En esta fase los implantes se han controlado clínicamente con sondaje, ortopantomografía y tomografía axial computarizada.

Los pacientes han acudido a la consulta cada 3 meses para la eliminación del aparato protésico y el correspondiente control clínico y radiológico. (Figs. 5 y 6 ).

Los parámetros que determinan el éxito de la intervención son los siguientes:

- Estabilidad del injerto.

- Estabilidad del implante.

- Ausencia de dolor.

- Ausencia de supuración.

- Contacto directo entre implante y hueso (comprobación radiológica).
- Reabsorción óseo-vertical menor de $1 \mathrm{~mm}$ en el primer año después de aplicación de la prótesis.

Todos los implantes han superado con éxito los 12 meses de aplicación implantoprotésica y se encuentran actualmente en una fase de seguimiento con una duración de 4 a 38 meses (con una media de 12,3 meses).

\section{RESULTADOS}

Todos los injertos han demostrado una buena estabilidad y se ha conseguido un volumen suficiente para la terapia implantoprotésica. Ha sido realizada la téc-

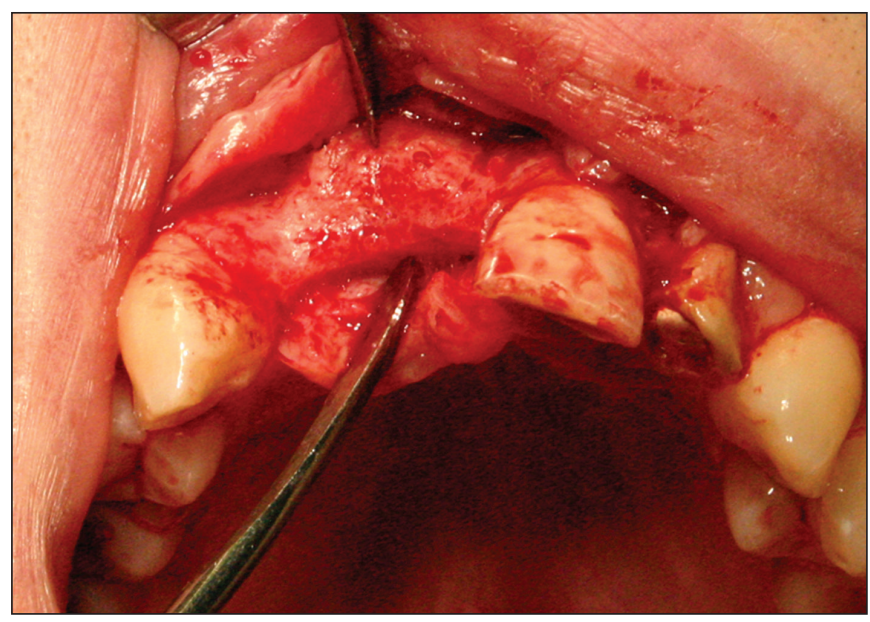

Fig. 5: Imagen intraoperatoria en la cual resulta evidente el aumento de espesor en el vestíbulo-palatino.

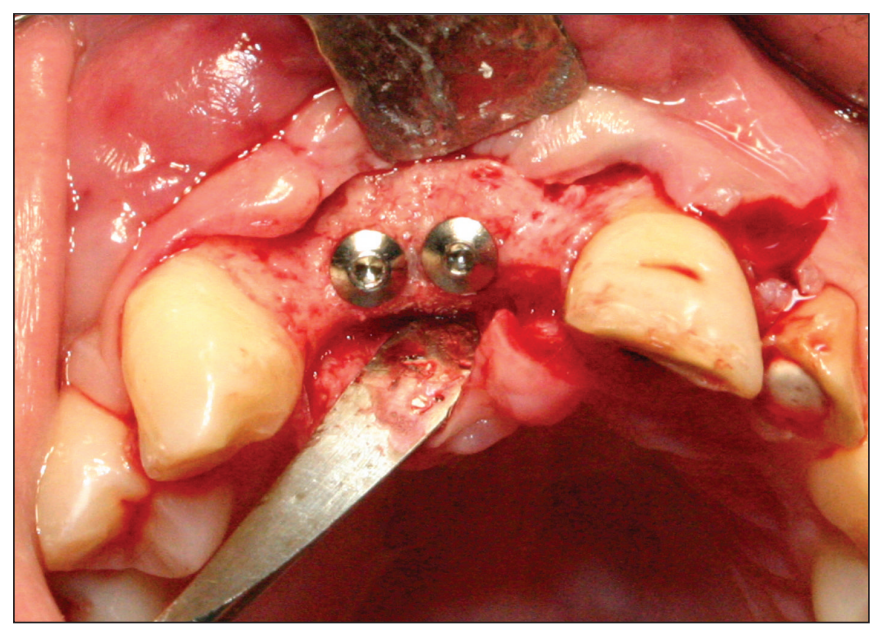

Fig. 6: Imagen intraoperatoria de la inserción de las fixture. 
nica de 2 fases para colocar 18 implantes de $3,5 \mathrm{~mm}$ de diámetro, y 24 de $4 \mathrm{~mm}$ de diámetro, todos ellos de $10 \mathrm{~mm}$ de longitud. Todos han demostrado buena estabilidad primaria. Una vez transcurridos los 6/4 meses necesarios para realizar la osteointegración, no se ha perdido ningún implante y a los 12 meses todos los casos resultan haber sido protesizados con éxito.

La ganancia de la densidad ósea ha sido valorada realizando mediciones a partir de imágenes radiológicas de los maxilares (TAC) los tiempos considerados son:

- Antes de la intervención.

- Después de la colocación del injerto.

- Antes de la introducción de los implantes.

- Después de 12 meses de la aplicación implantoprotésica.

Los valores medios de aumento en la arcada maxilar (Tabla 2) con el injerto son de 7,63 mm en fase posoperatoria, de $6,45 \mathrm{~mm}$ en el momento de la introducción de los implantes (15,47\% de pérdida) $6,18 \mathrm{~mm}$ a los 12 meses de la aplicación implantoprotésica ( $19 \%$ de pérdida respecto a la fase de injerto).

Los valores medios de aumento en la arcada mandibular (Tabla 3) con el injerto son de $7 \mathrm{~mm}$ en fase poso- peratoria, 5,85 mm en el momento de introducir los implantes ( $16,43 \%$ de pérdida) medida que se ha mantenido a los 12 meses de la aplicación implantoprotésica.

Analizando los resultados obtenidos, resulta que en la arcada superior, aunque el aumento medio haya sido más conspicuo respecto de la arcada mandibular, se ha verificado no sólo una mayor reabsorción total del tejido óseo injertado, sino también una reducción de la dimensión transversal en la fase siguiente a la de introducción de los implantes, situación que no se produce en la arcada inferior.

Por lo que se refiere a las complicaciones postoperatorias a cargo de la zona donante y de la zona receptora, en 6 pacientes $(33,3 \%)$ se ha manifestado una disestesia pasajera en la zona de la extracción, resuelta en 4-6 meses, y una equimosis posoperatoria en 9 pacientes $(50 \%)$. En ningún paciente se ha producido dehiscencia de las heridas ni se han manifestado síntomas de insensibilidad en el labio inferior, ni permanentes ni temporales.

Por lo que se refiere al manejo del dolor en fase posoperatoria, 4 pacientes $(22,22 \%)$ no han tenido que recurrir a antiinflamatorios no esteroides, 10 pacientes $(55,56 \%)$ han hecho uso de antiinflamatorios no este-

\section{TABLA 2.- CASUÍSTICA DE LAS INTERVENCIONES REALIZADAS EN EL MAXILAR SUPERIOR}

\begin{tabular}{|c|c|c|c|c|}
\hline Caso $^{\circ}$ & Espesor postinjerto & Espesor preimplante & $\begin{array}{c}\text { Densidad a los 12 meses de la } \\
\text { aplicación implanto-protésica }\end{array}$ & $\mathbf{N}^{\circ}$ implantes \\
\hline 1 & 8 & 6 & 6 & 2 \\
\hline 2 & 8 & 6 & 6 & 3 \\
\hline 5 & 8 & 7 & 6 & 3 \\
\hline 7 & 9 & 7 & 6 & 3 \\
\hline 8 & 9 & 7 & 7 & 3 \\
\hline 10 & 6 & 5 & 5 & 2 \\
\hline 12 & 6 & 5 & 5 & 3 \\
\hline 13 & 7 & 7 & 6 & 3 \\
\hline 15 & 8 & 7 & 7 & 3 \\
\hline 16 & 8 & 7 & 7 & 2 \\
\hline 18 & 7 & 7 & 7 & 3 \\
\hline Media & $7,63(0,979)$ & $6,45(0,79)$ & $6,18(0,7158)$ & 3 \\
\hline
\end{tabular}




\section{TÄLA 3.- CASUÍSTICA DE LAS INTERVENCIONES REALIZADĀS EN EL MAXILAR INFERIOR}

\begin{tabular}{|c|c|c|c|c|}
\hline Caso $\mathbf{n}^{\circ}$ & Densidad postinjerto & Espesor preimplante & $\begin{array}{c}\text { Densidad a los 12 meses de la } \\
\text { aplicación implanto-protésica }\end{array}$ & $\mathbf{N}^{\circ}$ implantes \\
\hline $3 *$ & 6 & 5 & 5 & 2 \\
\hline $4 *$ & 7 & 6 & 6 & 2 \\
\hline $6 *$ & 8 & 5 & 5 & 1 \\
\hline $9 *$ & 6 & 6 & 6 & 2 \\
\hline $11^{*}$ & 8 & 6 & 6 & 2 \\
\hline $14^{*}$ & 7 & 6 & 6 & 2 \\
\hline $17^{*}$ & 7 & 7 & 7 & 2 \\
\hline Media & $7(0,75)$ & $5,85(0,6389)$ & $5,85(0,6389)$ & \\
\hline
\end{tabular}

roides durante menos de 3 días, mientras que 4 pacientes $(22,22 \%)$ los han utilizado más de 3 días y menos de 7 .

\section{DISCUSIÓN}

La finalidad de la experimentación es ofrecer los datos clínicos de la metodología utilizada en la Clinica di Odontoiatria dell'Università degli Studi di MilanoBicocca (Clínica de Odontología de la Universidad de Milán-Bicocca) para el tratamiento de los edentulismos de IV clase, según Cawood y Howell (1), intercalados en ambos maxilares. El programa terapéutico prevé una primera intervención para obtener el aumento de espesor transversal de la cresta mediante un injerto de hueso autólogo extraído de la sínfisis mentoniana, seguido, a los 6 meses de distancia, de otra intervención para la colocación de implantes y finalmente de la rehabilitación implantoprotésica.

Se han propuesto distintos procedimientos para obtener el aumento de la cresta alveolar:

- Injertos de hueso autólogo de origen intraoral, fijados mediante la colocación de implantes (Jensen \& Sindet-Pedersen 1991) (7).

- Bone chips y membranas, seguidas en un segundo momento de la inserción de los implantes (Buser y otros 1996; Nevins \& Mellonig 1994) $(8,9)$.

- Bone chips y membranas posicionadas en la misma intervención de introducción de los implantes (Simion y otros 1998) (10).
De los resultados obtenidos resulta evidente que el hueso autólogo está especialmente indicado para obtener un aumento de grosor óseo. En particular, la sínfisis mentoniana ha demostrado ser la zona preferida, respecto a otras zonas intra y extra orales, para tratar este tipo de atrofias, en función del volumen necesario y de la sencillez del tratamiento de la fase operatoria.

Los resultados ponen de manifiesto que este tipo de metodología da lugar a resultados altamente previsibles, con una reducción media de volumen del hueso neoformado de un $17,41 \%$ respecto al volumen del injerto colocado, y que la remodelación del hueso en la arcada superior parece estar más marcada respecto de la arcada mandibular. Además, en fase de prótesis y seguimiento, se ha manifestado una alta estabilidad dimensional del hueso neoformado y una osteointegración idéntica a la que se manifiesta en tejido no tratado con una muestra autóloga.

Esto nos permite afirmar que, para el tratamiento de las atrofias de IV clase, la extracción de hueso autólogo de la sínfisis mentoniana constituye el estándar de referencia para el éxito terapéutico.

\section{BIBLIOGRAFÍA}

1. Cawood JI, Howell RA. A classification of the edentulous jaws. Int J Oral Maxillofac Surg. 1988 Aug; 17(4):232-6.

2. Cordaro et Al. Alveolar ridge augmentation with mandibular bone blocks. Clin Oral Impl Res. 2002;13:103-11. 
3. Misch CM, Misch CE, Resnik RR, Ismail YH. Reconstruction of maxillary alveolar defects with mandibular symphysis graft for dental implants: a preliminary and procedural report. International Journal of Oral and Maxillofacial Implants. 1992;7:360-6.

4. Misch CM. Comparison of intraoral donor sites for onlay grafting prior to implant placement. International Journal of Oral and Maxillofacial Implants. 1997;12:767-76.

5. Zins JE, Whittacker L.A. Membranous versus endochondral bone autografts: implications for craniofacial reconstruction. Plastic and Reconstructive Surgery. 1983 72:778-86.

6. Phillips JH, Rhan BA. Fixation effects on membranous and endochondral onlay bone graft revascularization and bone deposition. Plastic and Reconstructive Surgery. 1990;85:891-7.

7. Jensen J, Sindet-Pedersen S. Autogenous mandibular bone grafts and osseointegrated implants for reconstruction of the severely atrophied maxilla: a preliminary report. Journal of Oral and Maxillofacial Surgery. 1991;49:1277-87.
8. Buser D, Dula K, Hirt HP, Schenk RK. Lateral ridge augmentation using autografts and barrier membranes: clinical study with 40 partially edentulous patients. Journal of Oral and Maxillofacial Surgery. 1996;54: 420-32.

9. Nevins R, Mellonig JT. The advantages of localized ridge augmentation prior to implant placement: a staged event. International Journal of Periodontology and Resorative Dentistry. 1994;14:97.

10. Simion M, Jovanovic SA, Trisi P, Scarano A, Piattelli A. Vertical ridge augmentation around dental implants using a membrane technique and autogenous bone or allograft. International Journal of Periodontology and Resorative Dentistry. 1998;18:8-23.

\section{CORRESPONDENCIA}

\author{
Gianluca Porcaro \\ Via Boito 115 \\ 20052 - Monza (MI). \\ 0039.347.30.39.301 \\ porcarogianluca@libero.it
}

